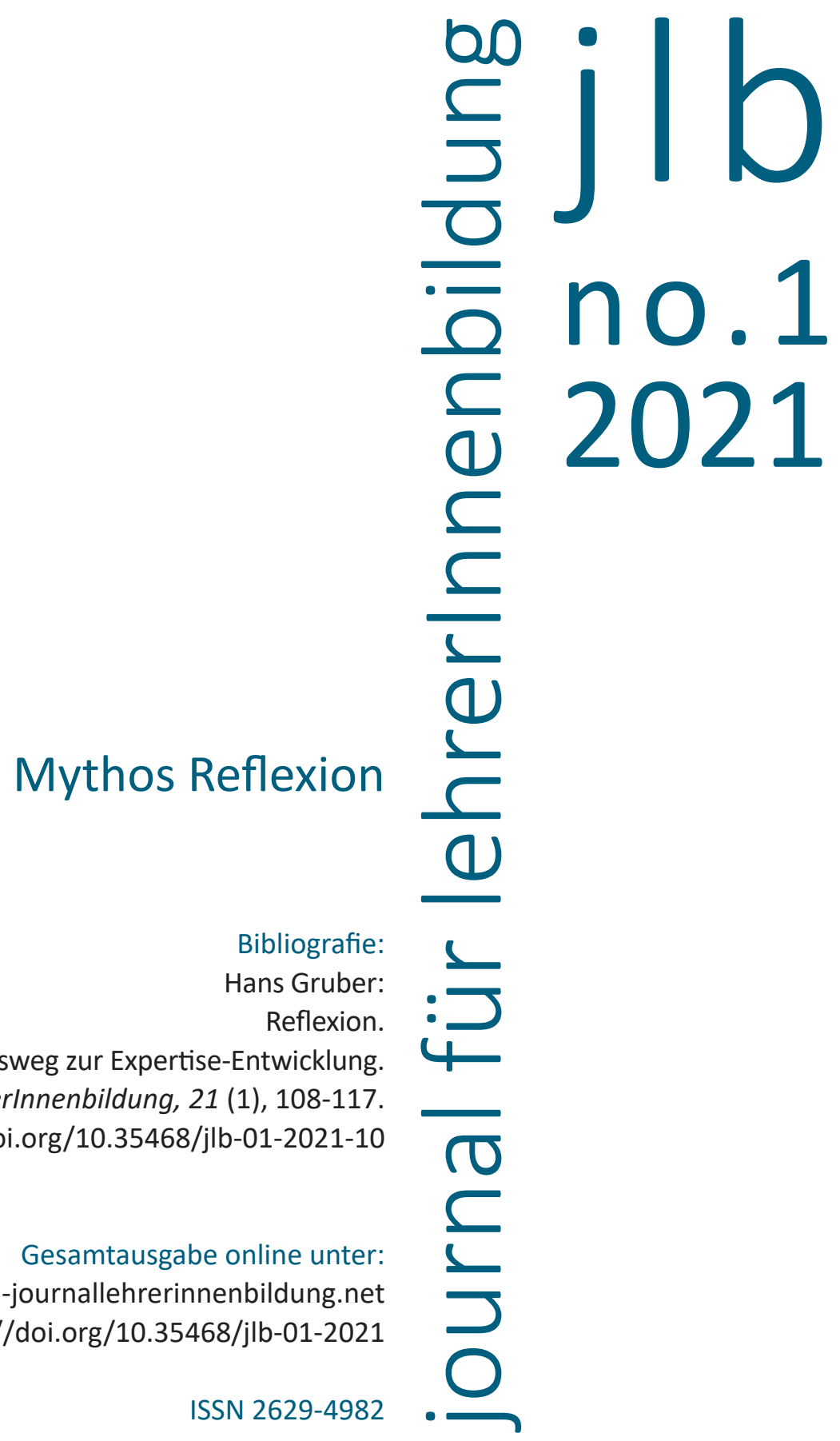


10

Hans Gruber

\section{Reflexion. Der Königsweg zur Expertise-Entwicklung}


Expert*innen sind auf der Sonnenseite des Lebens, was ihre berufliche Leistung angeht, denn sie (1) besitzen eine umfangreiche Wissensbasis, (2) haben reichhaltige berufliche Erfahrungen gemacht und gut genutzt, (3) können großen Problemlöseerfolg vorweisen, (4) erledigen ihre Arbeit effizient, (5) zeichnen sich durch eine niedrige Fehlerquote aus und (6) arbeiten mit hoher Präzision (Gruber, 1999a). Im Gegensatz dazu ist die Leistung von Noviz*innen schwächer und anfälliger beziehungsweise bei gutem Erfolg eher zufällig und nicht einfach reproduzierbar. Pädagogisch wichtig ist, dass an der Verringerung des Leistungsunterschieds gearbeitet werden kann. Die geringere Leistungsfähigkeit von Noviz*innen resultiert aus Sicht der Expertiseforschung vor allem daraus, dass sie noch wenig spezifische Erfahrungen besitzen und demzufolge bislang zu wenig geübt haben und zu wenig Praxis vorweisen können, aus der sie die richtigen Schlussfolgerungen hätten ziehen können (Gruber \& Hascher, 2011).

Dem pädagogischen Optimismus, der hieraus spricht, da im Prinzip der Weg zur Expertise-Entwicklung allen offensteht, stehen allerdings vier Pferdefüße entgegen. Erstens dauert der Prozess in der Regel - zumindest in den meisten Berufen - recht lange (eine Faustregel spricht von zehn Jahren), zweitens müssen dabei aufwändige Lern- und Übeprozesse bewältigt werden, die spezifisch dem Ziel der Expertise-Entwicklung dienen, drittens müssen die in dem Prozess gemachten Erfahrungen über den gesamten Zeitraum subjektiv als relevant erkannt sowie angemessen und kritisch verarbeitet werden, und viertens erfordert dies alles in der Regel eine enge Koordination der Lernenden mit Personen, die den Entwicklungsprozess begleiten und steuern (Lehrkräfte, Trainer*innen, Mentor*innen usw.).

Berücksichtigt man sowohl die Charakteristika von Expert*innen, die in empirischen Studien in vielen verschiedenen Domänen herausgearbeitet werden konnten, als auch die Merkmale erfolgversprechender Expertise-Entwicklungsprozesse, wird ersichtlich, weswegen im Titel dieses Beitrags Reflexion als Königsweg bezeichnet wurde. Im Verlauf der Expertise-Entwicklung ist es notwendig, große Mengen an - oft akademisch formuliertem, beispielsweise in Studiengängen oder Berufsausbildungen niedergelegtem - kanonischem Wissen zu erwerben und verfügbar zu halten, dies gleichzeitig in Handlungsabläufen zu erproben, zu validieren und auf den Prüfstand zu stellen, immer komplexeren Handlungsanforderungen gerecht zu werden, dabei sowohl gelingende Lernprozesse als solche zu identifizieren als auch die Ursa- 
chen von Fehlern und Missgeschicken zu erkunden, um sie in Zukunft vermeiden zu können, und zugleich innovativ die eigene Domäne voranzubringen. Dies alles stellt in quantitativer Hinsicht (etwa Umfang des Wissens, Dauer des Entwicklungsprozesses) erhebliche Anforderungen, erfordert zugleich aber fortwährende Integrationsprozesse, in denen unterschiedliche - positive wie negative, erwartungskonforme wie überraschende - Erfahrungen zusammengebracht werden müssen. Bei dieser Integrationsaufgabe spielt Reflexion eine herausragende Rolle, da sie die Gewähr bietet, dass die angesprochenen unterschiedlichen Komponenten ineinanderwirken können.

Bevor diese Rolle in drei Teilschritten skizziert wird, sind noch einige Gedanken zur Konzeption des Begriffs Reflexion nötig, die nach von Wright (1992) mit Reflections on reflection überschrieben werden.

\section{Reflections on reflection}

Die Eigenschaft eines Spiegels, eintreffende Lichtstrahlen zu reflektieren, erlaubt es dem Menschen, einen Blick auf sich selbst zu werfen. Im übertragenen Sinn meint Reflexion zunächst „Wiedergabe“ beziehungsweise „Widerspiegelung" von Information, im weiteren Sinn das Nachdenken über sich selbst. Dementsprechend unterscheidet von Wright (1992) zwei Ebenen von Reflexion, die beide für Lern- und Erziehungsprozesse bedeutsam, aber sorgfältig zu unterscheiden sind. Auf der einen Ebene bezieht sich Reflexion auf die äußere Welt, auf der anderen Ebene auf die eigene Person. Beide Varianten sind in die Kategorie der higher-order processes einzuordnen, es handelt sich um metakognitive Formen von Wissen und Fertigkeiten, mit denen eine "widerspiegelnde" Analyse kognitiver Elemente der Informationsverarbeitung vorgenommen wird. Von Wright (1992) nutzt eine Expertise-Metapher, um die beiden Ebenen von Reflexion zu charakterisieren: „Novice[s] (...) master a wide variety of routine events and can, in one sense of the word, reflect on them." (S. 60) Darin bilden sich metakognitive Fertigkeiten ab, die auf die äußere Welt gerichtet sind - somit Reflexion einer Person über Gegenstände, Sachverhalte und Prozesse in ihrer Umgebung. Was hierin nicht enthalten ist, ist Reflexion über die eigene Person als intentionales Subjekt eigener Handlungen, die Expert*innen kennzeichnet: 
„Reflecting about one's own knowledge or intentions involves an element which is absent from reflections about the surrounding world. Self-reflection presupposes, in the language of mental models, a ,metamodel': in order to reason about how I reason, I need access to a model of my reasoning performance." (S. 61)

Reflexion im Sinn der Monitorings und der metakognitiven Analyse eigenen Handelns erfordert also, dass die Person zurücktritt, das eigene Handeln interpretiert und daraus Schlüsse zieht. Von Wright (1992) weist darauf hin, dass sich insbesondere konstruktivistische didaktische und pädagogische Ansätze mit dem Training dieser Form der Reflexion beschäftigten, etwa reciprocal teaching oder cognitive apprenticeship.

\section{Reflexion als Indikator der Bewertung von Wissensbeständen}

Die Fähigkeit von Expert*innen, große, bedeutungsvolle Muster in Material aus ihrer Domäne wahrzunehmen, sich rasch einzuprägen und zuverlässig zu erinnern, hat mit der Organisation des Wissens zu tun, die Kriterien inhaltlicher Relevanz folgt, so dass das Wissen funktional und effizient zur Problemlösung eingesetzt werden kann. Paradoxerweise sind daher bei Expert*innen die Anforderungen an Prozesse der Gedächtnissuche trotz der größeren Wissensbasis geringer, so dass mehr Freiraum für die erfolgreiche Bewältigung aktueller Probleme zur Verfügung steht (Gruber, 1999b).

Die Gedächtnisinhalte, über die Expert*innen verfügen, sind eng mit Anwendungs- und Handlungsmöglichkeiten verknüpft, wie bereits Chase und Simon (1973) in ihrer pattern recognition-Theorie postulierten. Gobet und Simon (1996) verfeinerten die Theorie der Mustererkennung um das Konzept der templates, das Suchmechanismen in komplexen Datenstrukturen postuliert, die dazu dienen, dass Informationsrepräsentationen von Expert*innen kontinuierlich evaluiert und episodisch eingebettet werden. Die enge Verknüpfung von Bewertungs- und Wissensverarbeitungsprozessen war bereits Kernstück im SEEK-Modell (Search, EvaluatE, and Know; Holding, 1985); dabei spielen Evaluations- und Reflexionsprozesse eine zentrale Rolle.

Ein besonders aussichtsreicher, aber schwer zu fassender pädagogischer Anwendungsbereich solcher Verknüpfungen ist im Umgang mit 
Fehlern zu finden. Die Komplexität beruflicher Anforderungen führt unweigerlich dazu, dass auch Expert*innen - wenngleich selten - Fehler begehen. Fehler sind einerseits unerfreulich, haben andererseits aber das Potenzial der Thematisierung wertbezogenen Handelns und Urteilens und besitzen daher großen Stellenwert für die Entwicklung und Aufrechterhaltung von Expertise. Dies erfordert allerdings Reflexion über die Frage der Verantwortung einzelner Menschen für von ihnen begangene Fehler. In der Theorie des negativen Wissens (Oser, Hascher \& Spychiger, 1999) wurde herausgearbeitet, dass zu den konstruktiven Strategien, die die Arbeit mit Fehlern besonders fruchtbar machen, die offene Ursachensuche und die Reflexion und Bewertung von Erfahrung zählen.

\section{Reflexion als Teil von Lern- und Übeprozessen in der Expertise-Entwicklung}

Die enge Verknüpfung von Wissen und Bewertungen, die Expert*innen kennzeichnet, stellt beträchtliche Anforderungen an pädagogische Versuche, Expertise-Entwicklung zu unterstützen. Die einschlägigen Ansätze stimmen - auch wenn sie teils unterschiedliche Terminologie verwenden, da sie verschiedene Phasen der Entwicklung adressierendarin überein, dass es eines langen Zeitraums gezielter Übung bedarf, in dem die eigenen Lern- und Arbeitserfahrungen immer wieder rückwirkend analysiert werden, um Fehler oder Verbesserungsmöglichkeiten zu entdecken. Dabei ist die Unterstützung durch Dritte (Lehrkräfte, Trainer*innen, Ausbilder*innen usw.) oft vonnöten, um neuartige Entwicklungschancen in Angriff nehmen zu können. Dies wird etwa im Ansatz des cognitive apprenticeship (Collins, Brown \& Newman, 1989) thematisiert, der darauf aufbaut, dass Expert*innen die Lernenden mittels sequenzierter didaktischer Methoden unterweisen: Modellierung (Vormachen einer Vorgehensweise durch die Expert*innen mit Erklärungen) - Coaching (Beobachten der Lernenden bei der Ausführung, Feedback) - scaffolding (Unterstützungsmaßnahmen, die sich auf einzelne Teile der Aufgabe beziehen) - Ausblenden instruktionaler Maßnahmen. Die Lernenden gewinnen zunehmend Autonomie im Handeln, indem sie zu Artikulation (eigenes Wissen artikulieren und diskutieren), Reflexion (eigene Vorgehensweise mit denjenigen an- 
derer vergleichen und sich mit der eigenen Vorgehensweise kritisch auseinandersetzen) und Exploration (eigenes Vorgehen hinterfragen sowie Schwierigkeiten herausarbeiten) angeregt werden.

Ericsson, Krampe und Tesch-Römer (1993) führten das Konzept von deliberate practice ein; sie konnten zeigen, dass Expert*innen nicht nur über einen langen Zeitraum viel geübt hatten, sondern, dass sie vor allem gezielt geübt hatten, um ihre Leistungsfähigkeit zu verbessern. Deliberate practice bezeichnet eine Form von Übung, die darauf abzielt, die eigene Leistung zu verbessern; hierfür ist es erforderlich, dass der kritischen Bewertung und Reflexion des eigenen Handelns großer Wert geschenkt wird, um Fehler oder Verbesserungsmöglichkeiten zu entdecken. Besonders interessante Beispiele zu deliberate practice stammen aus der Musik oder dem Profisport (Pietsch \& Gruber, 2017). Da die dritten Personen oft im Hintergrund agieren, prägten Gruber, Lehtinen, Palonen und Degner (2008) für sie die Bezeichnung persons in the shadow. Solche Personen sind auch und gerade für Expert*innen notwendig, die schon hohen Expertisegrad erreicht haben, denn deren Schwächen sind oft schwer zu identifizieren. Zudem ist deren mögliche weitere Entwicklung anspruchsvoll, so dass sie die relevanten Punkte nicht selbst entdecken und sie nur mit Hilfe kontinuierlicher Reflexion durch Dritte bearbeiten können. Auch die Fehler, die Expert*innen begehen, sind oft komplex und kompliziert, so dass ihre fortwährende Analyse und die Konzeption von Gegenmaßnahmen besonders schwierig ist.

\section{Reflexion als gezielte Nutzung beruflicher Erfahrung}

Untersuchungen zur Expertise-Entwicklung zeigten, dass sich die im vorigem Abschnitt skizzierten Lern- und Übeprozesse regelhaft in komplexen Domänen zeigen. Während das Modell der deliberate practice auf die Merkmale erfolgversprechender Übeprozesse und deren Einbettung in pädagogische Planungs- und Unterstützungsmaßnahmen abzielt, stellen die Theorie der Wissensenkapsulierung (Boshuizen \& Schmidt, 1992) sowie fortfolgend die Theorie der Wissensrestrukturierung durch Fallbearbeitung (Boshuizen, Gruber \& Strasser, 2020) Versuche dar, die intraindividuellen Expertise-Entwicklungsprozesse 
zu beschreiben. In diesen Versuchen spielt die Reflexion im Sinne gezielter Nutzung beruflicher Erfahrung eine herausragende Rolle. Beide Theorien bauen auf Schema- und Skriptansätzen auf; bezogen auf Expertise-Entwicklung war das Modell des dynamischen Gedächtnisses (Kolodner, 1983) wegweisend. Darin wurde versucht zu erklären, wie Menschen ihre individuellen, subjektiv gefärbten Erfahrungen ertragreich für ihre weitere Expertise-Entwicklung nutzen können. Das Wissen von Expert*innen wird in dem Modell über episodische Definitionen beschrieben, die mit zunehmender Erfahrung fortgesetzt verfeinert werden, so dass es zu erfahrungsbasierter Wissenrestrukturierung kommt. Episodische Definitionen sind eine Sonderform von generalisiertem Wissen über Ereignisse und Handlungen, das erfahrungsabhängig verändert wird. Solche dynamischen Erfahrungswissenseinheiten (E-Mops: episodic memory organization packets) umfassen sowohl generalisierte Erfahrungen als auch Abweichungen erlebter Einzelepisoden davon. Regelhaftigkeiten und Abweichungen werden durch den fortgesetzten reflexiven Umgang mit Erfahrung erlangt, wobei wiederkehrende Ähnlichkeiten erlebter Episoden den generalisierbaren Anteil stärken, während Fehler und Abweichungen gesondert indiziert und erinnert werden.

Studien vor allem aus der Medizin lieferten die Initiative für die Entwicklung der Theorie der Wissensenkapsulierung. Es zeigten sich enorme Unterschiede in den diagnostischen und therapeutischen Problemlöseprozessen und Entscheidungen in Abhängigkeit vom Expertisegrad der Mediziner*innen. Expertise-Entwicklung geht offenbar mit qualitativen Veränderungen der Wissensbasis aufgrund intensiver, reflexiv verarbeiteter Nutzung der beruflichen Erfahrung einher. Während zu Beginn der Karriere (etwa im Praktischen Jahr) diagnostische Entscheidungen vornehmlich auf zuvor erworbenem biomedizinischem Wissen gründen, wird in der weiteren beruflichen Entwicklung immer mehr auf klinisches Wissen Bezug genommen. Expert*innen schließlich können - unter scheinbarem Verzicht auf grundlegendes Wissen - fallbasiert agieren (Boshuizen \& Schmidt, 1992). Dabei ist aber zu beachten, dass es sich bei den Fallrepräsentationen nicht um idiosynkratisches Patient*innenwissen handelt, sondern um generalisierte Episoden im Sinne der Theorie des dynamischen Gedächtnisses. Im Verlauf der Expertise-Entwicklung wurde das biomedizinische Wissen restrukturiert, in klinisches Erfahrungswissen integriert, so dass es schnell und präzise in der enkapsulierten Form genutzt werden kann. Enkapsulierung 
bedeutet also Umwandlung von deklarativem Wissen in fallbezogenes Wissen aufgrund beruflicher Erfahrung. Boshuizen et al. (2020) führten aus, dass sich die entsprechenden Prozesse der Wissensrestrukturierung durch Fallbearbeitung auch in anderen Domänen, etwa dem Management (Arts, Gijselaers \& Boshuizen, 2006) oder der Psychotherapie (Caspar, 1995) und der Erziehungsberatung (Strasser \& Gruber, 2015), finden lassen. Auch dort spielt die Nutzung der Erfahrung mit einer Vielzahl von Fällen eine wichtige Rolle, und wieder ist es der reflexive Blick auf das eigene Handeln, der dabei bedeutsam ist.

Dass Reflexion als Teil beruflicher Leistung auch über die Bedeutung für die Expertise-Entwicklung hinaus für den beruflichen Alltag wichtig ist, konnte in einer Reihe von Studien gezeigt werden, in denen in verschiedenen Domänen signifikante und bedeutsame korrelative Zusammenhänge zwischen Reflexion und einer Reihe weiterer Merkmale nachgewiesen wurden, etwa mit Fehlerorientierung und Lernen aus Fehlern (Hetzner, Gartmeier, Heid \& Gruber, 2011) oder mit Veränderungsbereitschaft und persönlicher Initiative (Hetzner, Heid \& Gruber, 2012).

\section{Reflexion - ein Königsweg auch im Lehrberuf}

Erfahrung stellt also eine wesentliche Grundlage von Expert*innenHandeln in beruflichen Domänen dar und wird durch eine langwährende, reflexive, oft durch Dritte angeleitete Auseinandersetzung mit den Anforderungen des Berufs sowie das Erleben entsprechender Episoden nutzbar (Gruber, 1999a).

Aufgrund der subjektiven Bedeutsamkeit von Erfahrung und von erinnerten Inhalten für die Expertise-Entwicklung ist es naheliegend, dass die pädagogisch-psychologische Gestaltung von Lernbedingungen und Lernumgebungen, in denen die Nutzung von Erfahrung gefördert werden soll, in der Regel Rückgriffe auf Konzepte der Reflexion umfasst. Die entsprechenden Ansätze der Expertiseforschung lassen sich auch auf den Lehrberuf übertragen (Gruber \& Hascher, 2011). Um die Anforderungen des Lehrberufs erkennen, komplexe Unterrichtssituationen differenziert verstehen, Handlungsmöglichkeiten abwägen und den Unterricht sowie den Umgang mit Kindern und Jugendlichen angemessen gestalten zu können, bedarf es hoher Expertise (Berliner, 2001), die auf langjähriger reflektierter Praxis und Übung und einer ständig erneuerten und restrukturierten Wissensbasis gründet. 
Die Fähigkeit, im Klassenzimmer wichtige Muster schnell und präzise erfassen zu können, erleichtert es, anspruchsvolle sowie multiple Ziele im Unterricht zu verfolgen, wirkungsvolle, auch individualisierte Lerngelegenheiten für die Schüler*innen bereitzustellen und für ein gutes Klassenklima zu sorgen. Expertise im Sinn der Enkapsulierung umfasst sowohl das Beherrschen von Automatismen und Routinen im Unterricht als auch die Sensitivität für die Besonderheit spezifischer Situationen und Schüler*innen. Damit Expertise-Entwicklung gelingt, ist die gerichtete Nutzung von Erfahrung und die Unterstützung durch Lehrkräfte, Trainer*innen, Mentor*innen oder Expert*innen über einen langen Zeitraum notwendig.

In diesem Sinne kann Reflexion dann zum Königsweg der Entwicklung und Aufrechterhaltung von Expertise werden, wenn sie zielgerichtet ist und durch Dritte begleitet wird, damit sie auch zielführend wird.

\section{Literatur}

Arts, J. A. R., Gijselaers, W. H. \& Boshuizen, H. P. A. (2006). Understanding managerial problem-solving, knowledge use and information processing: Investigating stages from school to the workplace. Contemporary Educational Psychology, 31(4), 387410. https://doi.org/10.1016/j.cedpsych.2006.05.005

Berliner, D. C. (2001). Learning about and learning from expert teachers. International Journal of Educational Research, 35(5), 463-482.

Boshuizen, H. P. A., Gruber, H. \& Strasser, J. (2020). Knowledge restructuring through case processing: The key to generalise expertise development theory across domains? Educational Research Review, 29. https://doi.org/10.1016/j.edurev. 2020.100310

Boshuizen, H. P. A. \& Schmidt, H. G. (1992). On the role of biomedical knowledge in clinical reasoning by experts, intermediates and novices. Cognitive Science, 16(2), 153-184. https://doi.org/10.1207/s15516709cog1602_1

Caspar, F. (1995). Information processing in psychotherapy intake interviews. In B. Boothe, R. Hirsig, A. Helminger, B. Meier \& R. Volkart (Hrsg.), Perzeption - Evaluation - Interpretation (S. 3-10). Bern: Hogrefe \& Huber.

Chase, W. G. \& Simon, H. A. (1973). The mind's eye in chess. In W. G. Chase (Hrsg.), Visual information processing (S. 215-281). New York: Academic Press.

Collins, A., Brown, J. S. \& Newman, S. E. (1989). Cognitive apprenticeship: Teaching the crafts of reading, writing, and mathematics. In L. B. Resnick (Hrsg.), Knowing, learning, and instruction (S. 453-494). Hillsdale: Erlbaum.

Ericsson, K. A., Krampe, R. T. \& Tesch-Römer, C. (1993). The role of deliberate practice in the acquisition of expert performance. Psychological Review, 100(3), 363-406.

Gobet, F. \& Simon, H. A. (1996). Templates in chess memory: A mechanism for recalling several boards. Cognitive Psychology, 31(1), 1-40.

Gruber, H. (1999a). Erfahrung als Grundlage kompetenten Handelns. Bern: Huber. 
Gruber, H. (1999b). Wie denken und was wissen Experten? In H. Gruber, W. Mack \& A. Ziegler (Hrsg.), Wissen und Denken. Beiträge aus Problemlösepsychologie und Wissenspsychologie (S. 193-209). Wiesbaden: Deutscher Universitäts-Verlag. https://doi.org/10.1007/978-3-663-08225-5_10

Gruber, H. \& Hascher, T. (2011). Lehrer/innenexpertise zwischen Wissen und Können. In S. Rahm \& C. Nerowski (Hrsg.), Enzyklopädie Erziehungswissenschaft Online (EEO), Fachgebiet Schulpädagogik. Weinheim: Juventa. https://doi.org/10.3262/ EEO09110173

Gruber, H., Lehtinen, E., Palonen, T. \& Degner, S. (2008). Persons in the shadow: Assessing the social context of high abilities. Psychology Science Quarterly, 50(2), 237-258.

Hetzner, S., Gartmeier, M., Heid, H. \& Gruber, H. (2011). Error orientation and reflection at work. Vocations and Learning: Studies in Vocational and Professional Education, 4(1), 25-39. https://doi.org/10.1007/s12186-010-9047-0

Hetzner, S., Heid, H. \& Gruber, H. (2012). Change at work and professional learning: How readiness to change, self-determination and personal initiative affect individual learning through reflection. European Journal of Psychology of Education, 27(4), 539-555. https://doi.org/10.1007/s10212-011-0094-1

Holding, D. H. (1985). The psychology of chess skill. Hillsdale: Erlbaum.

Kolodner, J. L. (1983). Towards an understanding of the role of experience in the evolution from novice to expert. International Journal of Man-Machine Studies, 19(5), 497-518.

Oser, F., Hascher, T. \& Spychiger, M. (1999). Lernen aus Fehlern. Zur Psychologie des negativen Wissens. In W. Althof (Hrsg.), Fehlerwelten: Vom Fehlermachen und Lernen aus Fehlern (S. 11-41). Opladen: Leske + Budrich.

Pietsch, S. \& Gruber, H. (2017). Wann glaubt man der Autoritätsperson "Trainerin" und warum? In A. Bernholt, H. Gruber \& B. Moschner (Hrsg.), Wissen und Lernen. Wie epistemische Überzeugungen Schule, Universität und Arbeitswelt beeinflussen (S. 215-234). Münster: Waxmann.

Strasser, J. \& Gruber, H. (2015). Learning processes in the professional development of mental health counselors: Knowledge restructuring and illness script formation. Advances in Health Sciences Education, 20(2), 515-530. https://doi.org/10.1007/ s10459-014-9545-1

Von Wright, J. (1992). Reflections on reflection. Learning and Instruction, 2(1), 59-68.

Hans Gruber, Prof. Dr. phil. Dr. h. c. für Pädagogik an der Universität Regensburg. Arbeitsschwerpunkte: Expertiseforschung, Eye-Tracking, Netzwerkanalyse

hans.gruber@ur.de

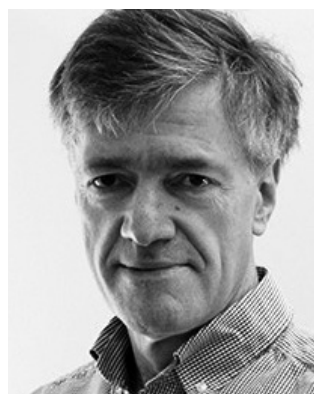

